\title{
Telehealth using ECG Sensor and Accelerometer
}

\author{
Hristijan Gjoreski*, Aleksandra Rashkovska**, Simon Kozina*, Mitja Luštrek*, Matjaž Gams*, \\ * Department of Intelligent Systems, Jožef Stefan Institute, Ljubljana, Slovenia \\ ${ }^{* *}$ Department of Communication Systems, Jožef Stefan Institute, Ljubljana, Slovenia \\ \{hristijan.gjoreski, aleksandra.rashkovska, simon.kozina, mitja.lustrek, matjaz.gams\}@ijs.si
}

\begin{abstract}
The increasing size of the elderly population is driving the development of ambient assisted living systems and telehealth. The recognition of the user's everyday activities and detection of alarming situations are important components of such systems. Moreover, the monitoring of vital signs, like the ECG, has a key role in telecare and telemonitoring systems. Therefore, in this paper we propose a system that monitors the user by combining an ECG sensor and two accelerometers. Our system recognizes the user's activities and detects falls using the accelerometer data. The ECG data is analyzed in order to extract relevant physiological signals: heart rate, respiration rate, etc. In order to improve the reliability and robustness of the system, the measured accelerometer signals can be combined with the ECG signal in order to detect anomalies in the user's behavior and heart-related problems. The proposed proof-of-concept system could contribute significantly to the quality, unobtrusiveness and robustness of the health care and patient safety.
\end{abstract}

\section{INTRODUCTION}

The world's population is aging rapidly, threatening to overwhelm the society's capacity to take care of its elderly members. The percentage of persons aged 65 or over in developed countries is projected to rise from $7.5 \%$ in 2009 to $16 \%$ in 2050 [1]. This is driving the development of innovative ambient assisted living (AAL) technologies to help the elderly live independently for longer and with minimal support from the working-age population $[2,3$, 4]. To provide timely and proper assistance, AAL systems must understand the user's situation and context, making activity recognition (AR) an essential component $[5,6,7]$. Fall detection (FD) is an important component of many AAL systems because almost half of the hospitalizations of the elderly are caused by falls [8]. Fear of falling is an important cause for nursing home admission [9], and "the long lie" (not being able to get up and call for help) is an important predictor of death within six months [10].

The introduction of modern information and communication technologies (ICT), as support to medical activities, can increase the efficacy of the health care system and decrease its costs. Numerous studies have confirmed the benefit of the development of telecare systems [11, 12]. The proposed mobile approaches to telecare rely on body sensor networks that collect and manage recorded vital data [13].

This work was partly supported by the Slovene Human Resources Development and Scholarship funds and partly by the CHIRON project - ARTEMIS Joint Undertaking, under grant agreement No. 2009-1100228. The authors acknowledge the financial support from the state budget by the Slovenian Research Agency under Grant P2-0095.
This paper presents a system that combines two subsystems that correspond to the type of the sensor-data they analyze: (i) electrocardiograph (ECG) sub-system, and (ii) accelerometer (ACC) sub-system.

The ECG sub-system is based on Wireless Bipolar Body Electrode (WBBE) [14] that captures and preprocesses body surface potential signals [15]. An additional feature is the ability to extract hidden information from the body surface potential, like the respiration rate [16], or combine synchronized data from three sensors for reconstruction of the standard 12-lead ECG $[17,18]$.

The ACC sub-system uses two 3-axial ACCs attached on the user's chest and right thigh. By using data analysis techniques it recognizes user's activities and detect falls in real-time. The architecture of the sub-system is based on: (i) rules, which detect falls, recognize postures (static activities) and ensure the behavior of the sub-system is predictable and robust, and (ii) classifiers trained with machine learning (ML) algorithms, to recognize dynamic activities, for which the rules are not sufficiently accurate. The ACC sub-system was evaluated at the EvAAL-2013 AR competition [19] and awarded the first place. The evaluation was performed in a living lab using several criteria: recognition performance, user-acceptance, recognition delay, system installation complexity, and interoperability with other systems.

The rest of this paper is organized as follows. First, the design of the prototype system is given with a brief functional description. Next, the sensors and the methods for analysis of the sensor data are described. The operation of the system is presented in the Experiments section. Finally, the conclusions are summarized and some directions for future work are given.

\section{SYSTEM DESCRIPTION}

The current prototype system (see Fig.1) consists of an ECG sensor and two wearable ACCs. The ECG sensor is placed on an appropriate position on the user's chest. The ACCs are attached on the user's chest and the right thigh using elastic Velcro straps. The data analysis is performed on a laptop using the raw sensors data acquired through Bluetooth. However, for the future implementation of the system, we are considering a smartphone implementation and combining the chest ACC and ECG sensor into a single device. Moreover, this device is planned to be multifunctional $[20,21]$, and beside the ECG and ACC data, should also measure: body temperature, body humidity, skin resistance, light, etc. 

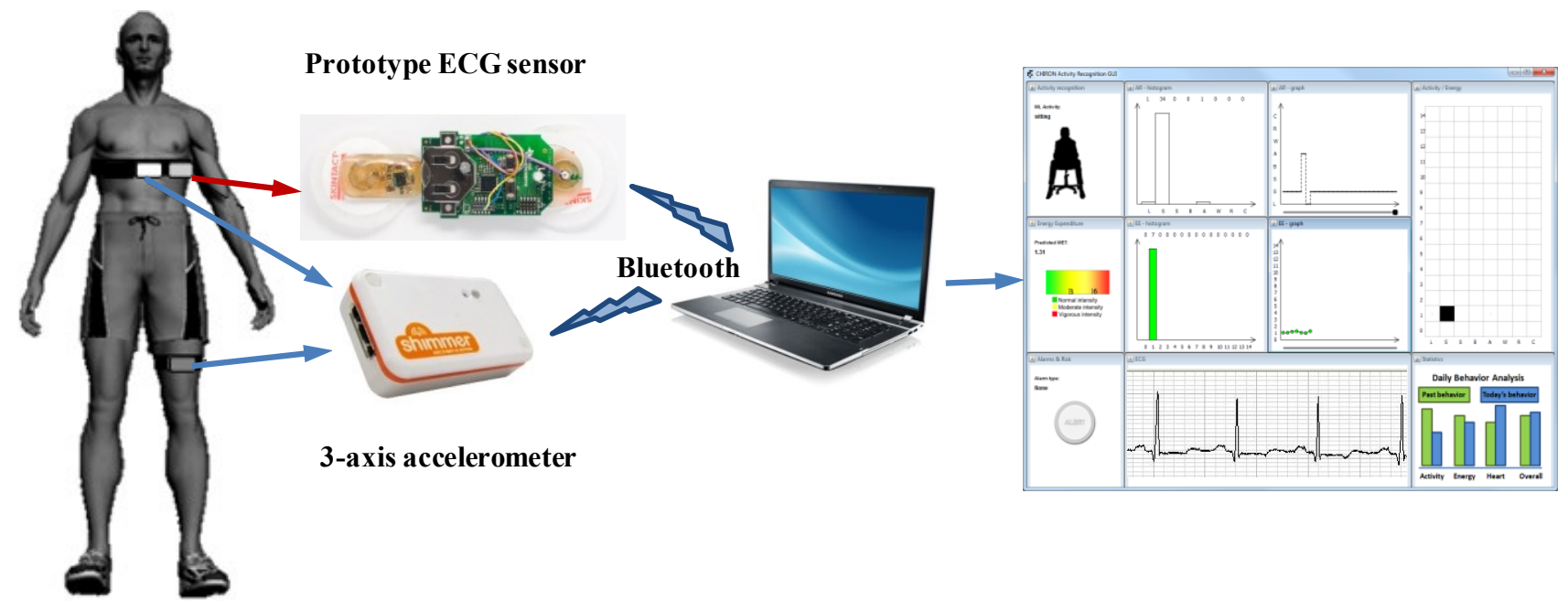

Figure 1. System design. Combination of the two solutions: ACC and ECG sub-systems.

\section{A. ECG sensor}

The ECG wireless body sensor designed from two self-adhesive electrodes positioned $5 \mathrm{~cm}$ apart, analog front-end, ultra-low-power (ULP) microcontroller and ULP Bluetooth V4.0 radio, ceramic chip antenna, and lithium coin battery, is shown in Fig.1 [22].

The analog front end is designed to suppress radio frequency interference. Signal amplification and filtering is made with a band-pass filter $(0.15 \mathrm{~Hz}$ to $250 \mathrm{~Hz},-3 \mathrm{~dB})$ before entering the microcontroller's 10 -bit A/D converter with sampling frequency of $125 \mathrm{~Hz}$ for monitoring, and up to $1000 \mathrm{~Hz}$ for diagnostic and research cases. The 16-bit microcontroller (Texas Instruments, MSP430F2274) has $32 \mathrm{kB}$ of FLASH, $1 \mathrm{kB}$ of RAM and on-chip temperature sensor, all with an average power in active mode of 270 $\mathrm{uA}$ at $1 \mathrm{MHz}$. The microcontroller has enough peripheral lines to support other on-board sensors, like 3-axis accelerometer, skin temperature sensor and others. The Nordic Semiconductor's chip nRF8001 was used to support Bluetooth radio connectivity in peripheral (slave) mode. We have chosen lithium coin battery CR2032 as a power source for the body sensor. The nominal cell capacity is $220 \mathrm{mAh}$ at discharge current of $200 \mathrm{uA}$, but due to pulse operation, a realistic capacity of $180 \mathrm{mAh}$ can be expected.

\section{B. Accelerometer}

An accelerometer (ACC) is a sensor that measures the acceleration applied to the sensor and also the constant Earth's gravity. When the ACC is at rest, only Earth's gravity is measured. Using this information, the velocity of the sensor and also the sensor orientation can be estimated and therefore used for AR and FD. The platform used in this study has a 3-axis ACC, uses Bluetooth communication, and has $2 \mathrm{~GB}$ of storage, which is enough to store 3 months of sensor data for offline analysis. However, for the needs of this study, only real-time data acquisition and analysis was used. A potential, more indepth, offline analysis is considered for future work.

\section{METHODS}

\section{A. ECG Data Analysis}

The measurement of ECG with sufficiently high amplitude and time resolution can support, besides standard heart rate analysis, also heart rate variability analysis [23], repolarization variability [24], ST-segment denivelation [25], detection of atrial fibrillation [26], characterization of arrhythmias, syncopes [27], sleep apneas, etc. Further, advanced approaches enable obtaining additional information from the recorded sensor signals. Two of them are listed below.

\section{1) Extraction of hidden information from $\mathrm{WBBE}$}

It is known that body surface potentials include a lot of information that can be used for the estimation of personal medical status. As a successful case, we refer to the respiration rate extraction directly from WBBE [16]. We confirmed that several DLs on positions near the center of the chest provide adequate signals for ECG-derived respiration (EDR) algorithms that can reliably extract respiration rates from variations in the R-peak amplitudes. The EDR techniques, relevant to our work, are based on the observation that the positions of WBBEs on the chest surface move relative to the heart. Additionally, the transthoracic impedance varies as the lungs fill and empty. The proposed methodology is accurate enough for most practical cases and therefore useful for mobile health applications based on body sensors.

We have shown that a single WBBE is enough for the extraction of the respiration rate. However, if more electrodes are available, they can be applied to improve accuracy and robustness of the EDR results.

\section{2) Synchronized WBBEs on strategic positions}

The 12-lead ECG is the gold standard in cardiology and lies at the center of the decision pathway for the evaluation and management of patients. However, the conventional 12-lead ECG is obtained from ten electrodes connected with wires to a data collecting device. Its application therefore imposes obtrusion and has negative effects on patient's comfort. Moreover, due to the fact that the standard positions of the precordial electrodes are 
often difficult to locate accurately, particularly in seriously damaged patients, the application of the conventional 12lead ECG device can be impractical.

We have shown [17] that a small number of WBBEs as few as three - can reliably reproduce a standard 12-lead ECG and are, therefore, suitable for real wireless applications. The proposed approach can be personalized in the sense that optimal positions of sensors and transformation matrices can be calculated for each individual [18].

\section{B. Accelerometer Data Analysis}

The ACC sub-system pipeline is shown in Fig.2. First, the sensors transmit the raw acceleration data over Bluetooth to the processing unit, i.e., laptop. The data from both sensors are then preprocessed: synchronized, filtered and segmented. Then the pipeline splits in two. On one side, the segmented data are transformed into feature vectors for the AR module, which recognizes the user's activity. On the other side, the FD module checks the acceleration for falls. If a fall pattern is recognized, the user's orientation is checked. If the orientation corresponds to lying, a fall is detected. Both the AR and FD modules are evaluating the user's situation every 250 milliseconds using the last 2 seconds of sensor data. For instance, if the current system time is denoted with $t$, the FD module evaluates fall events in the $[t-2 \mathrm{~s}, t-1 \mathrm{~s}]$ interval, and the $[t-1 \mathrm{~s}, t \mathrm{~s}]$ interval is used to check if the user's orientation corresponds to lying. If the fall event is detected and the orientation is correct, the reported activity is falling, otherwise the reported activity is computed with the AR module in the $[t-2 \mathrm{~s}, t \mathrm{~s}]$ interval. The system thus reports the user's activity and detects falls with a two-second delay.

In the following sections, the AR and FD methods are briefly described. More details can be found in our previous work, [28, 29, 30] for AR, and [28, 31] for FD.

\section{1) Activity Recognition}

In the AR module, the activities are recognized by a three-level scheme [30]. The AR scheme was developed after empirical analysis of the data, which showed that some activities (such as cycling) are better recognized by a classifier trained only to distinguish that particular activity from the others. Therefore, on the first level, the feature vector is fed into a classifier trained by the Random Forest ML algorithm to distinguish cycling from the other activities. If the activity is not classified as cycling, the feature vector is passed to the second level,

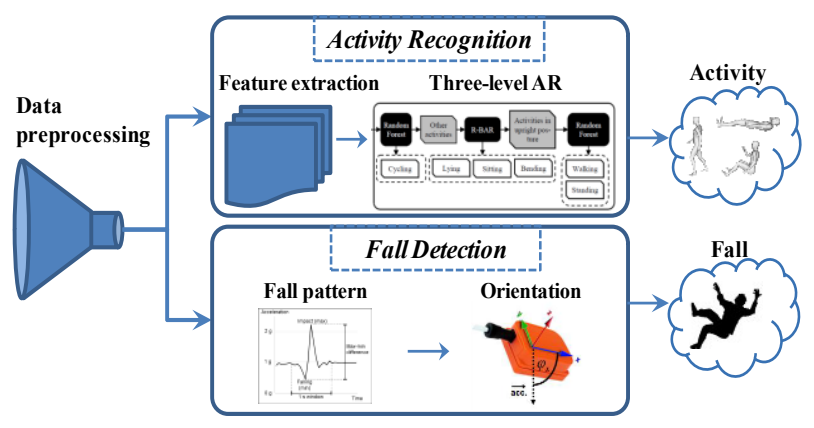

Figure 2. The data and recognition flow in the ACC sub-system. where the activities are recognized by rules. On this level, only the features that represent the sensor orientation the best are used (using component of the acceleration that corresponds to the gravity). The following activities are recognized at this level: sitting, lying, bending, and upright posture. If the recognized activity is the upright posture, the third level of $\mathrm{AR}$ is used to distinguish between standing and walking. The feature vector is fed into another Random Forest classifier, which is trained to separate these two activities.

\section{2) Fall Detection}

A typical acceleration pattern during a fall, measured by an ACC placed on the abdomen, is a decrease in acceleration followed by an increase [30]. This is because an ACC, when stationary, registers around $10 \mathrm{~m} / \mathrm{s}^{2}$ acceleration (denoted also as $1 \mathrm{~g}$ ), and during free fall 0 $\mathrm{m} / \mathrm{s}^{2}$ acceleration. When a person starts falling, the acceleration decreases from $1 \mathrm{~g}$ to around $0.5 \mathrm{~g}$ (perfect free fall is never achieved). Upon the impact with the ground, a short strong increase in the acceleration is measured.

To detect fall patterns, we used the length of the acceleration vector to ignore the direction of the acceleration. The minimum and the maximum acceleration within a one-second window were measured. If the difference between them exceeded $1 \mathrm{~g}$ and the maximum came after the minimum, a fall pattern was found. We augmented the fall-pattern detection with the measurement of the user's orientation after a potential fall. We assumed that the orientation of the user's body after a fall couldn't be upright. Therefore, a fall was detected if a fall pattern was detected and the orientation in the next second was not upright.

\section{EXPERIMENTS}

\section{A. ECG sensor performance results}

ECG signals from proximal electrodes have different appearance than the signals from standard ECG. An example is shown in Fig.3. The baseline is wandering and complete disappearance of the $\mathrm{P}, \mathrm{S}$ and $\mathrm{U}$ waves can be observed. Note that the $\mathrm{R}$ and $\mathrm{T}$ waves could have opposite polarity, which is rarely seen in standard ECG leads. It is evident that the position of the electrodes has a significant impact on the measured ECG signal. Detailed analyses are possible only after significant preprocessing targeted to specific goals, e.g. heart rate determination, arrhythmia identification and characterization, ST segment denivelation, etc.

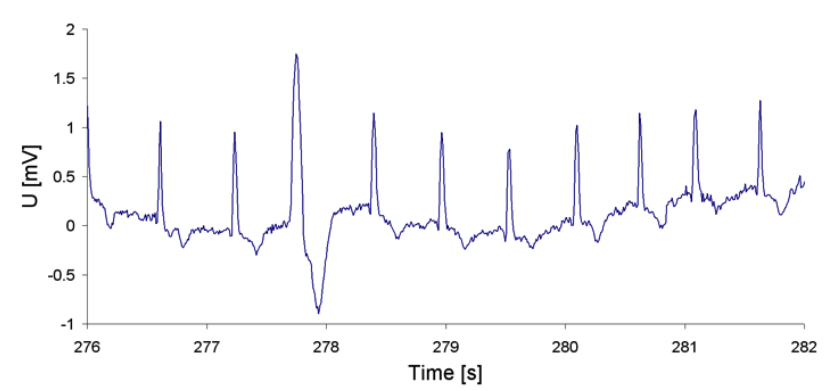

Figure 3. Raw ECG signal from electrodes at a distance of $5 \mathrm{~cm}$. The person is walking around. The third heart beat is a ventricular extra systole with large amplitude and prolonged timing of the QRST complex. 


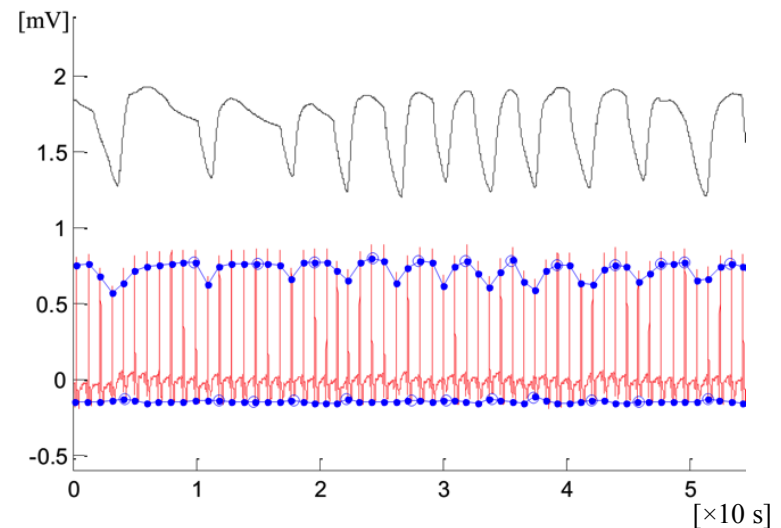

Figure 4. 53 seconds interval of an ECG (red) measured with a WBBE positioned in the chest center and 11 respiration intervals (black) measured by a thermistor near the front of the nose. 58 R-peaks (blue filled points) are detected and all 12 respiration interval are identified (blue circles).

For example, to determine the respiration rate, we found the best positions for the respiration body sensor [16] and proved that a single sensor of body surface potentials difference on proximal skin electrodes can be used for combined measurements of respiratory and cardiac activities (Fig.4).

\section{B. Activity Recognition \& Fall detection}

The evaluation of the ACC sub-system was performed in order to check the recognition performance of the methods, using a pre-recorded dataset. The evaluation was performed on a complex, 90-minute scenario, recorded from 10 people. The scenario was designed to capture the real-life conditions of a person's behavior, although it was recorded in a laboratory.

Table I shows the performance of the ACC subsystem. The performance of the AR is high, achieving $99.04 \%$ F-measure score averaged over all activities. The performance of the FD shows that $93.3 \%$ of the falls were detected (recall value), and $66.7 \%$ of all the fall detections were actually falls (precision value), giving the final $\mathrm{F}$ measure of $77.8 \%$. The detailed FD results (Table II) show that the first event - tripping (quick uncontrolled fall) was detected each time (15 out of all 15 events). The next event, fainting, was detected 13 out of 15 times. The next two events were the non-fall events that are difficult to distinguish from the fast falls because of the high acceleration. Because the FD module also checks the user's orientation after a potential fall, it was able to distinguish quickly sitting on the chair from the falls. However, this was not the case for quickly lying in the bed (13 detections - which are false because the event is non-fall). For correct recognition of this event, additional information would be needed, e.g., user's location [31].

\section{Concurrent ECG and Accelerometer Data}

Fig.5 shows activity experiment with concurrent measurements from ECG and ACC sensors when the user is lying, sitting and walking.

ACC sensors give clear information for distinguishing walking from sitting or lying. One can also notice lower beat rates in lying and sitting compared to walking, which implies eventual lower energy expenditure. Detailed analysis of ECG indicates also that beat rate of lying is
TABLE II. ACC SUB-SYSTEM-AR AND FD RESULTS

\begin{tabular}{l|l|l} 
Performance & Activity Recognition & Fall Detection \\
\hline Recall & $99.22 \%$ & $93.33 \%$ \\
\hline Precision & $98.85 \%$ & $66.67 \%$ \\
\hline F-measure & $99.04 \%$ & $77.78 \%$
\end{tabular}

TABLE I. FD DETAILED RESUlTS

\begin{tabular}{|c|c|c|}
\hline & Events & Detected/All \\
\hline \multirow{2}{*}{ 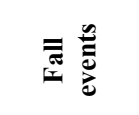 } & Tripping & $15 / 15$ \\
\hline & Fainting & $13 / 15$ \\
\hline \multirow{3}{*}{ 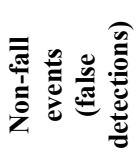 } & Quickly lying & $13 / 15$ \\
\hline & Quickly sitting & $1 / 15$ \\
\hline & Other & 0 \\
\hline
\end{tabular}

even lower that the one while sitting, which is supplementary information. With longer measurements, the long-term changes in the beat rate variability could give additional information about the time scale. Eventual extra systoles could point out a susceptibility to arrhythmia, etc.

\section{CONCLUSIONS}

We showed that the fusion of ECG and ACC sensors data can provide supplementary information about the status of the monitored user. Based on this, it is possible to better understand the context of user's health state and activity and therefore better reason about his/her health and behavior status. The reasoning may include classification of the detected anomaly into several levels: (i) low-risk warning, e.g., higher heart-rate detected during sedentary activity, (ii) medium-risk warning, e.g., gait anomaly detected, (iii) high-risk warning e.g., very high heart-rate detected during longer period of time, and (iv) alarming situation detected that requires medical attention, e.g., the user has fallen and is lying without movement.

We are currently developing and testing a new multifunctional sensor node that consists of several sensors to obtain synchronized data about vital bio-signs and activities of the monitored users. For the final implementation of the system we plan three-phase

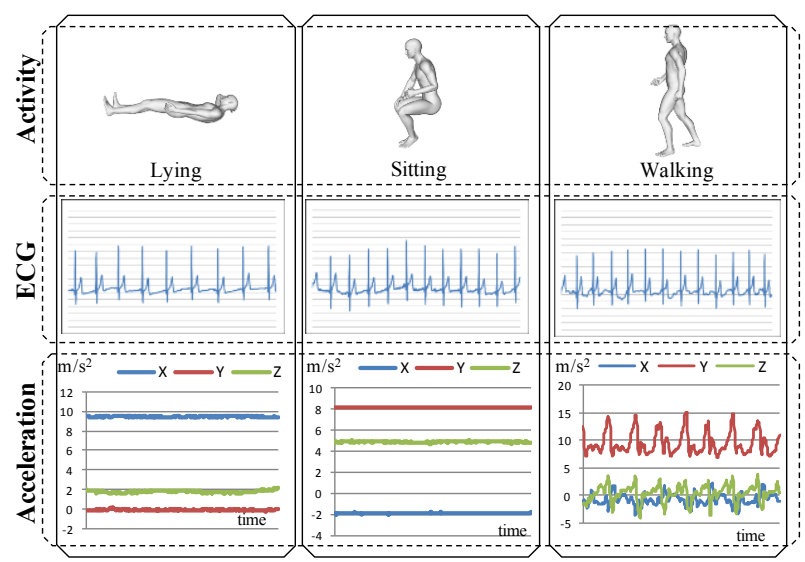

Figure 5. Concurrent ECG and ACC data 
monitoring and data analysis: (i) short-term behavior and health analysis - focusing on the last several minutes of data (alarming situations, falls, arrhythmias); (ii) mediumterm behavior analysis - focusing on the past day (gait analysis); and (iii) long-term behavior analysis (daily/weekly anomalies) related to heart problems, less active days, etc.

\section{REFERENCES}

[1] United Nations 2009, World population ageing, Report

[2] A. Bourouis, M. Feham, and A. Bouchachia, "A new architecture of a ubiquitous health monitoring system: a prototype of cloud mobile health monitoring system," The Computing Research Repository, 2012.

[3] M. Luštrek, B. Kaluža, B. Cvetković, E. Dovgan, H. Gjoreski, V. Mirchevska, and M. Gams, "Confidence: ubiquitous care system to support independent living" DEMO at European Conference on Artificial Intelligence, 2012, pp. 1013-1014

[4] B. Kaluža, B. Cvetković, E. Dovgan, H. Gjoreski, V. Mirchevska, M. Gams, and M. Luštrek, "A Multiagent care system to support independent living," International journal on artificial intelligence tools, 2014.

[5] D.A. Gregory, K. D. Anind, J. B. Peter, D. Nigel, S. Mark, and S. Pete, "Towards a better understanding of context and contextawareness," 1st International Symposium Handheld and Ubiquitous Computing, 1999, pp. 304-307.

[6] N. Vyas, J. Farringdon, D. Andre, and J. I. Stivoric, "Machine learning and sensor fusion for estimating continuous energy expenditure". Innovative Applications of Artificial Intelligence Conference, 2012, pp. 1613-1620.

[7] H. Gjoreski， B. Kaluža， M. Gams， R. Milić, and M. Luštrek "Ensembles of multiple sensors for human energy expenditure estimation," Proceedings of the 2013 ACM international joint conference on Pervasive and Ubiquitous computing, Ubicomp, 2013, pp. 359-362.

[8] M. J. Hall, L. Fingerhut, and M. Heinen, "National Trend Data on Hospitalization of the Elderly for Injuries, 1979-2001. American Public Health Association (APHA), 2004.

[9] M. E. Tinetti and C. S. Williams, "Falls, Injuries Due to Falls, and the Risk of Admission to a Nursing Home," The New England Journal of Medicine, 1997, pp. 1279-1284.

[10] D. Wild, U. S. Nayak, and B. Isaacs, "How dangerous are falls in old people at home?," British Medical Journal (Clinical Research Edition), vol. 282, no. 6260, 1982, pp. 266-268.

[11] A.G. Ekeland, A. Bowes, and S. Flottorp, "Effectiveness of telemedicine: A systematic review of reviews," Int. J. Med. Inform., vol. 79, 2010, pp. 736-771.

[12] W. Chaboyer, L. Thalib, M. Foster, C. Ball, and B. Richards, "Predictors of Adverse Events in Patients After Discharge From the Intensive Care Unit," Am. J. Crit. Care, vol. 17, 2008, pp. 255263.

[13] R. Trobec and U. Stanič, "Telehealth: A myth or reality?", Proceedings of MEET \& GVS on the 34th International Convention MIPRO 2011, Opatija, Croatia, 2011, pp. 295-300.

[14] R. Trobec, M. Depolli, and V. Avbelj, "Wireless network of bipolar body electrodes," Proceedings of the 7th International Conference on Wireless On-demand Network Systems and Services, WONS 2010, pp. 145-149.
[15] R Trobec, "Computer analysis of multichannel ECG," Computers in Biology and Medicine, vol. 33 , pp. 215-226, 2003.

[16] R.Trobec, A. Rashkovska, and V. Avbelj, "Two proximal skin electrodes - a respiration rate body sensor," Sensors, vol. 12, 2012, pp. 13813-13828.

[17] R. Trobec and I. Tomašić, "Synthesis of the 12-lead electrocardiogram from differential leads," IEEE Trans. Inf. Technol. Biomed., vol. 15, 2011, pp. 615-621.

[18] I. Tomašić, S. Frljak, and R. Trobec, "Estimating the Universa Positions of Wireless Body Electrodes for Measuring Cardiac Electrical Activity", IEEE Transactions on Biomedical Engineering, vol. 60, 2013, pp. 3368-3374.

[19] EvAAL competition. http://evaal.aaloa.org/ [Accessed: November, 2013]

[20] R. Trobec, V. Avbelj, and A. Rashkovska, "Multi-functional Wireless Body Sensor", Proceedings of GLOBAL HEALTH 2012: The First Internacional Conference on Global Health Challenges, 2012, pp. 71-74

[21] R. Trobec, V. Avbelj, and A. Rashkovska, "Multi-functionality of Wireless Body Sensors", Transactions on Internet Reserch, vol. 10 (1), 2014.

[22] K. Bregar and V. Avbelj, "Multi-Functional Wireless Body Sensor Analysis of Autonomy," MIPRO 2013, 35th International Convention, Opatija, Croatia, May 20-24, 2013, pp. 346-349.

[23] J.M. Kalisnik, V. Avbelj, R. Trobec, and B. Gersak, "Positiondependent changes in vagal modulation after coronary artery bypass grafting," Comput. biol. med., vol. 37, 2007, pp. 14041408.

[24] A. Rashkovska and V. Avbelj, "Signal processing methods for ST variability assessment in ECG," MIPRO 2013, 35th International Convention, Opatija, Croatia, May 20-24, 2013, pp. 355-358.

[25] A. Smrdel and F. Jager, "Automated detection of transient STsegment episodes in 24h electrocardiograms," Med. biol. eng. comput., vol. 42, 2004, pp. 303-311.

[26] B. Geršak et al., "European experience of the convergent atrial fibrillation procedure: Multicenter outcomes in consecutive patients", J Thorac Cardiovasc Surg., Early publication Aug. 2013.

[27] V. Avbelj and R. Trobec, "A closer look at electrocardiographic P waves before and during spontaneous cardioinhibitory syncope," International journal of cardiology, vol. 166, 2013, pp. e59-e61.

[28] H. Gjoreski, M. Luštrek, and M. Gams, "Accelerometer Placement for Posture Recognition and Fall Detection," In The 7th International Conference on Intelligent Environments, 2011, pp. $47-54$.

[29] S. Kozina, H. Gjoreski, M. Gams, and M. Luštrek,"Three-layer Activity Recognition Combining Domain Knowledge and Metaclassification," Journal of Medical and Biological Engineering, vol 33, no 4, 2013.

[30] S. Kozina, H. Gjoreski, M. Gams, and M. Luštrek, "Efficient Activity Recognition and Fall Detection Using Accelerometers," Evaluating AAL Systems Through Competitive Benchmarking Communications in Computer and Information Science, Volume 386, 2013, pp 13-23.

[31] H. Gjoreski, M. Luštrek, and M. Gams, "Context-Based Fall Detection using Inertial and Location Sensors," International Joint Conference on Ambient Intelligence, Lecture notes in computer science, 2012, pp. 1-16. 